\section{Evaluación de la calidad del agua en los canales de la Zona de Reglamentación Especial de Los Pantanos de Villa (Lima, Perú)}

Edith M. Enrique-Ayala ${ }^{*}{ }^{\oplus}$, Celina R. Gavidia Adriano ${ }^{2}{ }^{\oplus}$, Alvaro M. Garriazo Suni ${ }^{3 \oplus}$ y Daniela Changanaqui Alfaro ${ }^{4}$
Evaluation of the water quality in the channels within the Special Regulation Zone of Los Pantanos de Villa wetland (Lima, Peru)
1 Facultad de Ingeniería y Gestión, Universidad Nacional Tecnológica de Lima Sur. Lima, Perú. 2 Facultad de Ciencias, Universidad Nacional Agraria La Molina. Lima, Perú.

3 Facultad de Ciencias Ambientales, Universidad Científica del Sur. Lima, Perú.

4 Autoridad Municipal de Los Pantanos de Villa. Lima, Perú.

\section{SOUTH} SUSTAINABILITY

Citar como: Enrique-Ayala E. M. et al. (2021). «Evaluación de la calidad del agua en los canales de la Zona de Reglamentación Especial de los Pantanos de Villa (Lima, Perú)». South Sustainability, 2(2). e036. DOI: 10.21142/SS-0202-2021-e002

Artículo recibido: 14/11/2021 Revisado por pares

Artículo aprobado: 31/12/2021

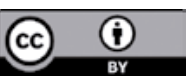

(c) Los autores, 2021. Publicado por la Universidad Científica del Sur (Lima, Perú)

*E-mail de correspondencia: miriam133114@gmail.com

\section{RESUMEN}

En la Zona de Reglamentación Especial de los Pantanos de Villa se ubican los canales Ganaderos 1 y Vista Alegre 2, que son primordiales por brindar un mantenimiento en los servicios ecosistémicos de provisión, como el agua para animales y el abastecimiento al humedal y la población de los pantanos. Sin embargo, el crecimiento poblacional y las actividades que se desarrollan en sus alrededores, como el vertimiento de aguas residuales, generan un impacto negativo en la calidad del agua. Por ello, el objetivo de la presente investigación es evaluar la calidad del agua de estos dos canales, mediante la comparación con las categorías 3 y 4 de los estándares de calidad ambiental (ECA) para agua y la determinación del índice de calidad del agua de la Unión Europea (ICA-UWQI). Los valores obtenidos en la medición de $\mathrm{pH}$, oxígeno disuelto y conductividad eléctrica son de 7,3, 3,3 gm/L y $62,1 \mu \mathrm{S} / \mathrm{cm}$, respectivamente; dicha data fue conseguida del promedio anual del periodo 2019-2021. Del monitoreo puntual se obtuvo que los parámetros de nitrato, fósforo, $\mathrm{DBO}_{5}, \mathrm{OD}$ y Escherichia coli no cumplen el ECA respectivo. Asimismo, el ICA-UWQI obtuvo un valor de 45,63, el cual tiene una clasificación marginal, es decir, se encuentra levemente impactado debido a la cercanía con la población y las fábricas.

Palabras clave: Pantanos de Villa, calidad de agua, parámetros fisicoquímicos, parámetros microbiológicos, metales tóxicos, servicios ecosistémicos

\section{ABSTRACT}

The Ganaderos 1 and Vista Alegre 2 channels are located in the Special Regulation Zone of Los Pantanos de Villa wetlands, and they are essential because they maintain provisioning ecosystem services, such as: water for animals, supply of the wetland and its population. However, population growth and the activities that occur in the surroundings, such as wastewater dumping, have a negative impact on water quality. Therefore, the aim of this research was to evaluate the water quality of these two channels, by comparing it with categories 3 and 4 of the Environmental Quality Standards (ECA) for water and the Universal Water Quality Index of the European Union (ICA-UWQI). The values obtained in the measurement of $\mathrm{pH}$, dissolved oxygen and electrical conductivity were 7.3, $3.3 \mathrm{gm} / \mathrm{L}$ and 62.1 $\mu \mathrm{S} / \mathrm{cm}$, respectively. This data was obtained from the annual average for the years 2019 to 2021. Through specific monitoring, it was found that the parameters of nitrate, phosphorus, BOD5, DO and Escherichia coli did not comply with the respective RCT. Also, the ICA-UWQI value obtained was 45.63 , which is a marginal classification; that is, slightly impacted due to proximity with the local population and factories.

Keywords: Los Pantanos de Villa wetlands, water quality, physicochemical parameters, microbiological parameters, toxic metals, ecosystem services. 


\section{Introducción}

Los humedales son ecosistemas que destacan en todo el planeta por los procesos hidrológicos y ecológicos, donde el agua es el principal factor regulador del medio, y es el componente hídrico necesario para que los ciclos de vida animal y vegetal se cumplan (Haustein, 2005). La importancia de los humedales radica en los servicios ecosistémicos que brindan, como la depuración del agua, los controladores de inundaciones, los reservorios de diversidad biológica, y permiten la recreación y el turismo (CONAF, 2013). Estudios señalan que los problemas de contaminación causados frecuentemente por metales pesados tienen un efecto silencioso, es decir, no se observan a simple vista; pero cuando nos damos cuenta del daño que producen, ya es tarde, debido a que son peligrosos para la salud (Romero, 2009). Las afecciones a la salud dependen del tipo de metal o metaloide, y pueden ser desde daños en órganos vitales hasta desarrollos cancerígenos (Combariza, 2009; Nava, 2011). Por otro lado, las altas concentraciones de agentes microbiológicos en el agua son originadas frecuentemente por cambios en el medioambiente y en la población, el desarrollo urbano no controlado, la inadecuada disposición de excretas humanas y animales, entre otros factores (Ríos, 2017), que causan efectos adversos a la salud ambiental y los servicios ecosistémicos.

Los refugios de vida silvestre proporcionan protección para diversos tipos de fauna y cobran mayor valor en entornos urbanos, debido a que contribuyen en la productividad de las comunidades o municipalidades al realizar actividades turísticas (Rodríguez et al., 2017). No obstante, la calidad del agua y la presencia de aves acuáticas se verán afectadas en la medida en que disminuyan las superficies de los humedales, a causa de actividades humanas inapropiadas que ocasionan impactos a estos ecosistemas (Aponte, 2015; Aponte et al., 2020), lo que provoca la reducción de la biodiversidad presente, ya que muchos organismos dependen de los humedales y de zonas ribereñas (Álvarez, 2016). En la costa peruana se ubica el Refugio de Vida Silvestre Los Pantanos de Villa (RVSPV), catalogado como sitio Ramsar, en el distrito de Chorrillos, en la ciudad de Lima Metropolitana. La zona presenta un área de 263,27 hectáreas y cuenta con 5 espejos de agua alimentados por manantiales y canales ubicados en la zona más baja del valle (Pulido, 2018). Sin embargo, en los últimos 10 años el RVSPV ha sufrido el fraccionamiento y la pérdida de su superficie a causa del crecimiento urbano, lo que provoca que el área natural se vea amenazada. Por otro lado, la ejecución e instalación de fábricas, lavanderías y camales a lo largo del área de este humedal viene afectando la calidad de sus recursos hídricos (Alvaro, 2020).

El abastecimiento de agua del humedal Pantanos de Villa se realiza a través de 7 canales y 3 manantiales, como los canales Ganaderos 1 y Vista Alegre 2. Actualmente, estos canales sufren impactos negativos debido a las descargas de aguas residuales causadas por las actividades recreativas, comerciales, de lavanderías y la falta de una red de desagüe (Raúl, 2011). Algunos canales presentan alta concentración de detergentes, que posteriormente afectan a la laguna Mayor, donde también se han registrado altos niveles de toxicidad para la vida acuática (Álvarez, 2016). No existen estudios que evalúen la calidad del agua de los canales de los Pantanos de Villa, y por ello no se conoce la capacidad que tiene este recurso para ser utilizado en función de sus características físicas, químicas y microbiológicas. En ese sentido, es importante conocer el estado de calidad del agua de estos canales, ya que puede convertirse en un vehículo transmisor de diversas enfermedades para los pobladores y una pérdida de recursos naturales.

El objetivo principal en esta investigación es evaluar la calidad del agua para los canales Ganaderos 1 y Vista Alegre 2 de los Pantanos de Villa, en función de parámetros fisicoquímicos $\left(\mathrm{pH}, \mathrm{T}^{\circ} \mathrm{C}, \mathrm{CE}\right.$ y $\left.\mathrm{SDT}\right)$, microbiológicos (coliformes totales, Escherichia coli [E. coli]) y metales tóxicos (plomo, mercurio, cadmio y arsénico). Además, se busca clasificar la calidad del agua mediante el índice de calidad del agua (ICA), el cual nos permitirá transformar los datos a una escala de medición única (Castro et al., 2014). Para esta investigación se aplicará el ICA-UWIQI desarrollado por el Consejo de la Unión Europea y Turquía (Boyacioglu, 2007).

\section{Materiales y métodos}

\section{Área de estudio}

El área de estudio son los canales Ganaderos 1 y Vista Alegre 2, ubicados en la periferia de la zona de Villa Baja del distrito de Chorrillos (figura 1). El muestreo se realizó

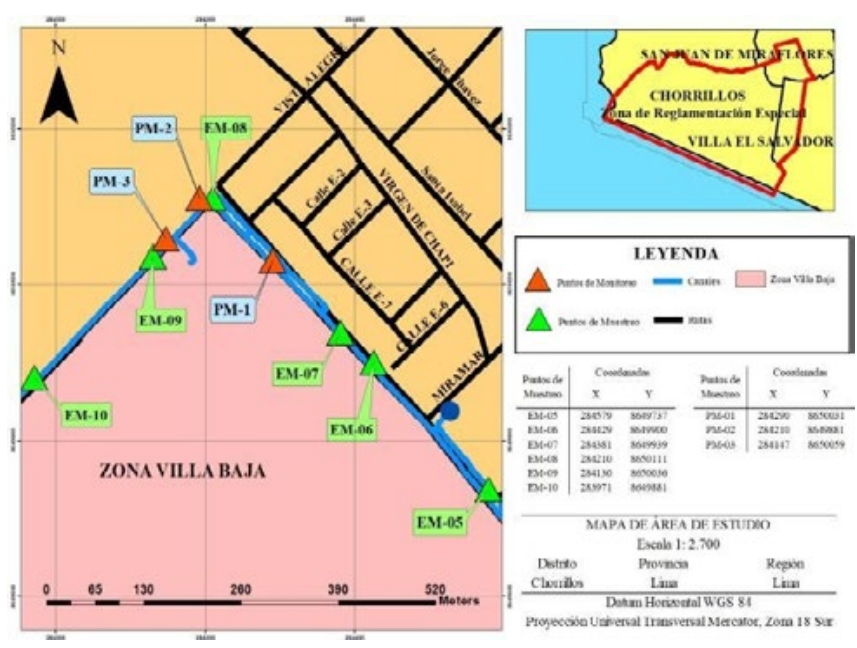

Figura 1. Mapa del área de estudio, donde se detallan los 3 puntos de muestreo (PM) y las 6 estaciones de monitoreo (EM) de los canales Ganaderos 1 y Vista Alegre 2. 
en abril de 2021, con base en el criterio del Protocolo Nacional para el Monitoreo de la Calidad de los Recursos Hídricos Superficiales de la ANA (2016). Se consideraron tres puntos de monitoreo superficiales con $20 \mathrm{~cm}$ de profundidad (figura 1). Previamente se llevó a cabo el reconocimiento del entorno, la rotulación y el etiquetado de los frascos para la toma de muestras.

\section{Parámetros de estudio}

La medición in situ de los parámetros fisicoquímicos como el potencial hidrógeno $(\mathrm{pH})$, temperatura $\left(\mathrm{T}^{\circ} \mathrm{C}\right)$, conductividad eléctrica (CE) y sólidos disueltos totales (SDT) se determinaron por medio de un multiparámetro Hanna HI 9813-6; para oxígeno disuelto (OD) se utilizó un oxímetro Hach Pocket Pro; para el fósforo total se utilizó un kit de prueba de ortofosfato $\mathrm{PO}-19$; y para el nitrato, un kit de nitrato Hach NI-11.

Asimismo, para la determinación de aceites y grasas (AcyG), detergente y demanda bioquímica de oxígeno $\left(\mathrm{DBO}_{5}\right)$ se recolectaron tres muestras por cada parámetro para ser llevadas al laboratorio acreditado CERTIMIN SA. La metodología empleada por el laboratorio varió para AcyG (SMEWW-APHA-AWWA-WEF. 23 rd Ed. 2017. Part5520 B), detergentes (SMEWW-APHA-AWWA-WEF. 23 rd Ed. 2017. Part-5540 C) y $\mathrm{DBO}_{5}$ (SMEWW-APHA-AWWAWEF. 23rd Ed. 2017. Part 5210 B).

La determinación de parámetros microbiológicos se realizó igualmente por medio del laboratorio acreditado CERTIMIN, y para ello se analizaron tres muestras por parámetro. La metodología empleada varió para coliformes fecales (termotolerantes) (SMEWW APHAAWWA-WEF 23 rd Ed.2017. Part-9221 E1) y Escherichia coli (E. coli) (SMEWW APHA-AWWA-WEF 23rd Ed. 2017. Part9221 F1).

La determinación de metales tóxicos se realizó también en el laboratorio acreditado CERTIMIN, y para ello obtuvieron tres muestras por parámetro. La metodología empleada para plomo, mercurio, cadmio y arsénico fue EPA Method 200.8 Rev. 5.4.1994. Determination of trace elements in waters and wastes by Inductively Coupled Plasma-Mass Spectrometry.

Es importante mencionar que para el análisis se recopiló la data histórica disponible (2019-2021), proporcionada por la Autoridad Municipal de Los Pantanos de Villa (Prohvilla), de monitoreos mensuales que se realizaron en seis estaciones de muestreo (EM) que pertenecen a los canales Ganaderos 1 y Vista Alegre 2. Se analizaron los parámetros de $\mathrm{pH}$ del periodo 2019-2021, el oxígeno disuelto (OD) y nitrato, ambos del periodo 2020-2021. Los datos fueron comparados con los valores establecidos del ECA para agua en la categoría 3 («riego de vegetales y bebida de animales»), subcategoría D1 («riego de vegetales»), subcategoría D2 («bebida de animales»), categoría 4 («conservación del ambiente acuático») y subcategoría E1 («lagunas y lagos») (Minam, 2017).

\section{Operacionalización de las variables}

Los valores obtenidos fueron comparados con los valores establecidos del ECA para agua en la categoría 3 («riego de vegetales y bebida de animales»), subcategoría D1 («riego de vegetales»), subcategoría D2 («bebida de animales») y categoría 4 («conservación del ambiente acuático»), subcategoría E1 («lagunas y lagos»), salvo el parámetro de solidos disueltos totales, ya que no se ha establecido en las categorías 3 y 4 del ECA para agua. Sin embargo, su análisis es importante para indicar la salinidad del agua; por ello, se ha considerado clasificarlo en agua dulce (SDT < 1000 ppm), agua salobre (1000 ppm $\leq$ SDT $\geq 5000$ ppm), agua altamente salobre (5000 ppm $\leq$ SDT $\geq 15000 \mathrm{ppm}$ ) y agua salina (15000 ppm $\leq$ SDT $\geq$ 30000 ppm) (Álvarez, 2015).

Además, se calculó el ICA-UWQI (Boyacioglu, 2007) a fin de mostrar el nivel de contaminación del agua de los canales Ganaderos 1 y Vista Alegre 2 a la fecha del muestreo. El valor del ICA se expresa en porcentajes; así, el agua contaminada asumirá un valor próximo o igual a 0 \% y el agua que presente óptimas condiciones obtendrá un valor cercano al 100. Los parámetros considerados para la aplicación de ICA-UWQI fueron $\mathrm{pH}, \mathrm{DBO}_{5}, \mathrm{OD}$, fósforo, nitrato, mercurio, cadmio y arsénico. La aplicación del ICA se realizó mediante la ecuación de suma ponderada y es la que se muestra en la ecuación 1 (Torres et al., 2009).

$$
\begin{aligned}
& n=1 \\
& \sum W_{i}^{*} I_{i} \ldots . \text { ecuación } 1 \\
& i=1
\end{aligned}
$$

Donde $W_{i}$ es el peso o porcentaje asignado al i-ésimo parámetro e $l_{i}$ es el subíndice del í-ésimo parámetro (Boyacioglu, 2007).

Los valores se expresan en porcentajes, a una escala de (0-100); es decir, en cinco categorías: pobre, entre 0 y 24 (se representan con un color rojo); marginal, entre $25 \mathrm{y}$ 49 (color naranja); regular, entre 50 y 74 (color amarillo); bueno, entre 75 y 94 (color verde); y excelente, entre 95 y 100 (color azul) (IDEAM, 2011).

\section{Resultados y discusión}

\section{Parámetros fisicoquímicos en los canales}

Los valores de $\mathrm{pH}$, detergente y aceites y grasa en los tres puntos de monitoreo, según los resultados, cumplen con la normativa de la categoría 3 y 4 del ECA para agua (tabla 1). Los valores del $\mathrm{pH}$ se encuentran entre un rango neutro y ligeramente alcalino. Los valores neutros del $\mathrm{pH}$ se deben a la gran diversidad de flora como las macrófitas; estas especies colaboran en la regulación de pH y evitan la acidez del agua (Baquero y Carrera, 2019). Asimismo, existe la posibilidad de que sean las micrófitas quienes regulen el $\mathrm{pH}$, ya que estas bordean los canales Ganaderos 1 y Vista Alegre 2 (Prohvilla, 2020). El análisis de detergentes en los tres puntos de monitoreo reportó 
un valor de 0,025 $\mathrm{mg} / \mathrm{L}$. La presencia de detergentes en el agua de los canales se debe al uso de lavandería que le da la población, y al vertimiento de aguas residuales e industriales (Pérez et al., 2017). Especies de macrófitas acuáticas como la Lemna spp y la Echorina spp remueven el sulfonato de alquilbenceno lineal, un componente usado para la fabricación de detergentes y productos de limpieza. Estas especies logran un porcentaje de remoción de más de 90 \% (Herrera, 2018). Los valores de aceites y grasas en los tres puntos de monitoreo son $<0,5 \mathrm{mg} / \mathrm{L}$. Su presencia no permite la reoxigenación mediante la interfase de aireagua, provoca la reducción del oxígeno disuelto del agua y absorbe la radiación solar, lo que afecta la actividad fotosintética de la flora acuática (ITSEMAP, 1994).

Los valores de la conductividad eléctrica (CE) y oxígeno disuelto (OD), según los resultados, sobrepasan los límites establecidos en la categoría 3 y 4 del ECA para agua. Los valores de la demanda bioquímica de oxígeno $\left(\mathrm{DBO}_{5}\right)$ en los tres puntos de monitoreo sobrepasan la categoría 4 del ECA para agua de $\geq 5 \mathrm{mg} / \mathrm{L}$. Los valores de PM-02 y PM03 de $\mathrm{DBO}_{5}$ cumplen con la categoría 3 del ECA para agua de $15 \mathrm{mg} / \mathrm{L}$, a excepción de PM-01 (tabla 1). Los valores de CE están relacionados con las fuentes de contaminación (Caho-Rodríguez y López-Barrera, 2017), ya que los canales reciben grandes cantidades de agua residual doméstica proveniente de las viviendas cercanas. Los altos valores de CE son producto de las impurezas presentes en el agua. Los valores de OD se encuentran por debajo de los $5 \mathrm{mg} / \mathrm{L}$ (tabla 1). Probablemente se deba a la saturación del suelo, que provoca ausencia de oxígeno, aunque haya un predominio de condiciones anaeróbicas (García et al., 2010). Ello podría afectar a los organismos que utilizan el oxígeno para el desarrollo de sus procesos vitales, como su respiración celular y adecuado crecimiento (ITSEMAP, 1994). No obstante, existen especies que pueden sobrevivir en condiciones escasas de oxígeno disuelto, como el guppy (Lebistes reticulatus) (Merino y Droste, 1983). La cantidad de $\mathrm{DBO}_{5}$ en los canales probablemente se deba a la presencia de algas, que generan más oxígeno de lo que ellas requieren para su respiración, ya que son parte de la cadena de alimentación de otras especies, como los guppies (Mara, 2003). Asimismo, en los cuerpos de agua ocurre que la materia orgánica en descomposición tiende a convertirse en materia inorgánica; al ser asimilada por algas, estas convierten la materia orgánica en descomposición en materia orgánica viva (Silva, 2004).

Los resultados indican que los valores de nitrato y fósforo sobrepasaron el límite establecido de $13 \mathrm{mg} / \mathrm{L}$ y $0,035 \mathrm{mg} / \mathrm{L}$, respectivamente, en la categoría 4 del ECA para agua (tabla 1). Los valores altos de nitrato probablemente se deban a las excretas de los animales como ovejas, cabras y perros, que se encuentran cerca de los canales. Los altos valores de nitrato generan problemas de toxicidad en organismos acuáticos y eutroficación en los cuerpos de agua (Cervantes-Carrillo et al., 2000). Cabe recalcar que las macrófitas intervienen en el proceso de oxidación por su capacidad de brindar oxígeno a través de sus raíces, mejorando la productividad microbiana aerobia y de tal forma la nitrificación (Domínguez, 2001). Las altas concentraciones de fósforo presentes en los canales Ganaderos I y Vista Alegre II probablemente se deban a los vertimientos de agua residuales domésticas e industriales, cargadas de detergentes, jabones y excretas de los animales, que causan eutroficación (Teixeira et al., 2013). De acuerdo con el resultado, los valores de sólidos disueltos totales (SDT) fueron mayores a 1999 ppm en los tres puntos de muestreo, lo que indica que el agua de los canales Ganaderos 1 y Vista Alegre 2 presentan agua de tipo salobre.

Los resultados señalan que los parámetros de detergentes, $\mathrm{pH}$, aceites y grasas presentan bajas concentraciones. Ello podría deberse a la presencia de la flora que bordean estos canales, como las macrófitas; Azolla filiculoides (helecho de agua), Hydrocotyle bonariensis (paragüita), Lemna minor (lentejita de agua) y algas. En el caso de las macrófitas, el proceso de fitodepuración ocurre cuando se produce el proceso de fotosíntesis, ya sean plantas acuáticas superiores o microscópicas (Fernández-Gonzales et al., 2005). Esto provoca una reducción de contaminantes a través de tres mecanismos: la filtración y sedimentación de sólidos, la incorporación de nutrientes en plantas y la degradación de materia orgánica por microorganismos que existen en las raíces de estas plantas (Martelo y Lara, 2012).

\section{Parámetros microbiológicos en los canales}

En la tabla 2 se presentan los resultados obtenidos de los tres puntos de muestreo para coliformes termotolerantes. Los puntos de muestreo PM-02 y PM-03 cumplen con la categoría 3 y 4 del ECA para agua, pero no es el caso del punto de muestreo (PM-01), debido a que presenta un valor mayor a $23 \mathrm{NMP} / 100 \mathrm{~mL}$. Los valores de Escherichia coli (E. coli) en los puntos de muestreo PM-01 y PM-03 presentan un valor mayor a $23 \mathrm{NMP} / 100 \mathrm{~mL}$ que sobrepasan el límite establecido en la categoría 3 del ECA para agua, a excepción del punto de muestreo (PM02), debido a que cumple con el límite establecido de 1000 NMP/100mL (tabla 2). Los agentes microbiológicos (coliformes termotolerantes y $E$. coli) son indicadores de contaminación patogénica y bacteriológica prevenientes de residuos fecales en el agua (Pérez et al., 2017; Reátegui, 2020). La presencia de los agentes microbiológicos en los canales Ganaderos 1 y Vista Alegre 2 se debe al vertimiento de aguas residuales y vertimiento de residuos sólidos que realiza la población y las fábricas aledañas. Sin embargo, se evidencia una baja concentración de coliformes termotolerantes (PM-02 y PM-03) que probablemente se deba a la presencia de un helecho acuático llamado Azolla filiculoides, ya que presenta una eficiencia de absorción del 72 \% según Solano Carrión (2019), que se encuentra en el transecto del canal Ganaderos I (Prohvilla, 2020). Según indica la Organización Mundial de la Salud (WHO, 2008), 
Tabla 1. Resultados de los parámetros fisicoquímicos de los canales Ganaderos 1 y Vista Alegre 2.

\begin{tabular}{|c|c|c|c|c|c|c|}
\hline \multirow{3}{*}{ Parámetros } & \multicolumn{2}{|c|}{$\begin{array}{l}\text { Categoría 3: riego de vegetales y bebida de } \\
\text { animales }\end{array}$} & \multirow{3}{*}{$\begin{array}{c}\begin{array}{c}\text { Categoría 4: } \\
\text { conservación del } \\
\text { ambiente acuático }\end{array} \\
\text { E1 } \\
\text { Lagunas y lagos }\end{array}$} & \multirow{3}{*}{$\begin{array}{c}\begin{array}{c}\text { Canal } \\
\text { Ganaderos } 1\end{array} \\
\text { PM-01 }\end{array}$} & \multirow{3}{*}{$\begin{array}{c}\begin{array}{c}\text { Canal } \\
\text { Ganaderos } 1\end{array} \\
\text { PM-02 }\end{array}$} & \multirow{3}{*}{$\begin{array}{c}\begin{array}{c}\text { Canal } \\
\text { Vista } \\
\text { Alegre } 2\end{array} \\
\text { PM-03 }\end{array}$} \\
\hline & D1 & \multirow{2}{*}{$\begin{array}{c}\text { D2 } \\
\begin{array}{c}\text { Bebida de } \\
\text { animales }\end{array}\end{array}$} & & & & \\
\hline & $\begin{array}{cc}\text { Agua para riego } & \text { Agua para riego } \\
\text { no restringido } & \text { restringido }\end{array}$ & & & & & \\
\hline $\mathrm{CE}(\mu \mathrm{S} / \mathrm{cm})$ & 2500 & 5000 & 1000 & 6490 & 6760 & 6850 \\
\hline $\mathrm{T}\left({ }^{\circ} \mathrm{C}\right)$ & - & - & - & 23,8 & 22,9 & 24 \\
\hline SDT. (PPM) & - & - & - & $>1999$ & $>1999$ & $>1999$ \\
\hline $\mathrm{pH}$ & $6,5-8,5$ & $6,5-8,4$ & $6,5-9,0$ & 6,9 & 7 & 6,8 \\
\hline Fósforo (mg/L) & - & & 0,035 & 0,4 & 0,2 & 0,06 \\
\hline Nitrato (mg/L) & - & - & 13 & 44 & 44 & 39.6 \\
\hline $\mathrm{DBO}_{5}(\mathrm{mg} / \mathrm{L})$ & 15 & 15 & 5 & 16,4 & 14,9 & 14,3 \\
\hline O.D. (mg/L) & $\geq 4$ & $\geq 5$ & $\geq 5$ & 3,3 & 4 & 3,8 \\
\hline Aceites y grasa (mg/L) & 5 & 10 & 5 & $<0,5$ & $<0,5$ & $<0,5$ \\
\hline Detergente (mg/L) & 0,2 & 0,5 & - & 0,025 & 0,025 & 0,025 \\
\hline
\end{tabular}

Tabla 2. Resultados de los parámetros microbiológicos de los canales Ganaderos 1 y Vista Alegre 2.

\begin{tabular}{|c|c|c|c|c|c|c|c|}
\hline \multirow{3}{*}{ Parámetros } & \multicolumn{3}{|c|}{$\begin{array}{l}\text { Categoría 3: riego de vegetales y bebida de } \\
\text { animales }\end{array}$} & \multirow{3}{*}{$\begin{array}{c}\begin{array}{c}\text { Categoría 4: } \\
\text { conservación del } \\
\text { ambiente acuático }\end{array} \\
\text { E1 } \\
\text { Lagunas y lagos }\end{array}$} & \multirow{3}{*}{$\begin{array}{c}\text { Canal } \\
\text { Ganaderos } \\
1 \\
\text { PM-01 }\end{array}$} & \multirow{3}{*}{$\begin{array}{c}\text { Canal } \\
\text { Ganaderos } \\
1 \\
\text { PM-02 }\end{array}$} & \multirow{3}{*}{$\begin{array}{c}\text { Canal Vista } \\
\text { Alegre } 2 \\
\text { PM-03 }\end{array}$} \\
\hline & \multicolumn{2}{|c|}{ D1 } & \multirow{2}{*}{$\begin{array}{c}\text { D2 } \\
\begin{array}{c}\text { Bebida de } \\
\text { animales }\end{array}\end{array}$} & & & & \\
\hline & $\begin{array}{l}\text { Agua para riego } \\
\text { no restringido }\end{array}$ & $\begin{array}{l}\text { Agua para riego } \\
\text { restringido }\end{array}$ & & & & & \\
\hline $\begin{array}{l}\text { Coliformes termotolerantes } \\
\text { (NMP/100 mL) }\end{array}$ & 1000 & 2000 & 1000 & 1000 & $>23$ & 23 & 23 \\
\hline $\begin{array}{l}\text { Escherichia coli } \\
\text { (NMP/100 mL) }\end{array}$ & 1000 & $* *$ & $* *$ & $* *$ & $>23$ & 23 & $>23$ \\
\hline
\end{tabular}

El símbolo ** dentro de la tabla significa que el parámetro no aplica para esta subcategoría.

la presencia de $E$. coli resulta alarmante, ya que puede causar graves infecciones transmitidas por el agua, como una gastroenteritis y diarrea grave que puede ser mortal para la población que hace uso de los canales como un recurso para sus actividades diarias (lavar ropa, bañarse, etc.).

\section{Metales tóxicos en los canales}

En la tabla 3 se muestran los resultados obtenidos en los tres puntos de muestreo para los parámetros de plomo, cadmio, mercurio y arsénico, los cuales cumplen con la categoría 3 y 4 del ECA de agua por sus bajos valores de concentración. De acuerdo con lo previsto, la ausencia de estos metales podría deberse al mecanismo de remediación que presentan las plantas acuáticas en los humedales al absorber o acumular los contaminantes en sus raíces (Delgadillo et al., 2010). Esto es concordante con la presencia de vegetación en los alrededores de los canales; entre ella destaca la presencia de especies macrófitas como la Azolla filiculoides, que se caracteriza por depurar aguas contaminadas por compuestos inorgánicos y orgánicos, su tolerancia a diferentes metales (como el cobre, el plomo, el arsénico, entre otros), y su rápido desarrollo de reproducción (Sánchez, 2010). Esta propiedad natural hace posible que no se vea reflejado el impacto observado en los canales por vertimiento de efluentes domésticos que presentan restos metabólicos, jabones y detergentes, y contribuyen con cantidades regulares de metales como el cromo, zinc y plomo (Correa, 2014). De igual modo, la presencia de desmontes y residuos sólidos, entre ellos los residuos fluorescentes, bombillas eléctricas (RAEE), que contienen trazas de mercurio, no influyen de manera significativa en la calidad del agua (Fajardo, 2018).

Nuestros resultados no evidenciaron la presencia de metales, lo cual resulta óptimo para la salud de los pobladores. Sin embargo, es fundamental mencionar 


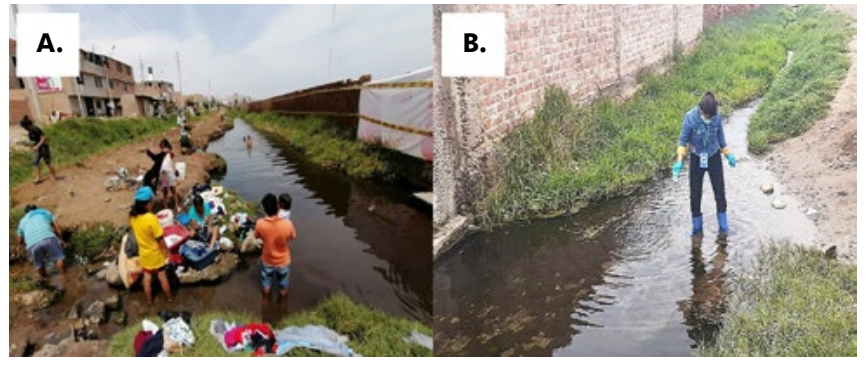

Figura 2. a) La población de Villa Baja utiliza el canal Ganaderos 1. b) Monitoreo en el canal Vista Alegre 2.

que muchos contaminantes que ingresan a las aguas superficiales suelen quedarse asentados en los sedimentos del fondo del cauce, lo que provoca efectos tóxicos sobre el sistema acuático (Espitia, 2014). Así lo muestra el estudio realizado en el humedal El Paraíso, que determina alta concentración de plomo, cadmio, mercurio y arsénico en los sedimentos (Ruiz, 2013). Por la tanto, para asegurar la ausencia de los metales y el riesgo que puedan presentar los pobladores se tendrá que realizar un análisis en los sedimentos del canal.

\section{Análisis de data histórica}

En la figura 3 se observa que la variación temporal del $\mathrm{pH}$ de 6,8-7,4 cumple con la categoría 3 del ECA para agua de $6,5-8,5$ y la categoría 4 de conservación del ambiente acuático de 6.5-9. Los valores del pH presentan una similar tendencia neutra para ambos canales y en casi todo el periodo de análisis de estudio, a excepción de los meses de febrero y julio, cuando se refleja una mayor acidificación, lo cual podría deberse al menor uso del agua por parte de los pobladores. El valor casi constante del $\mathrm{pH}$ se debe probablemente al mayor uso de los servicios ecosistémicos, ya sea para actividades de lavandería, limpieza de carro o bebida de animales (Álvarez, 2016). Además, el empleo de detergentes domésticos incrementa la concentración de fosfatos, lo que genera un aumento de nutrientes $y$, con ello, de $\mathrm{pH}$ (Gaxiola, 2010).

En la figura 4 se observa que la variación del oxígeno disuelto en ambos canales discrepa con el ECA para las

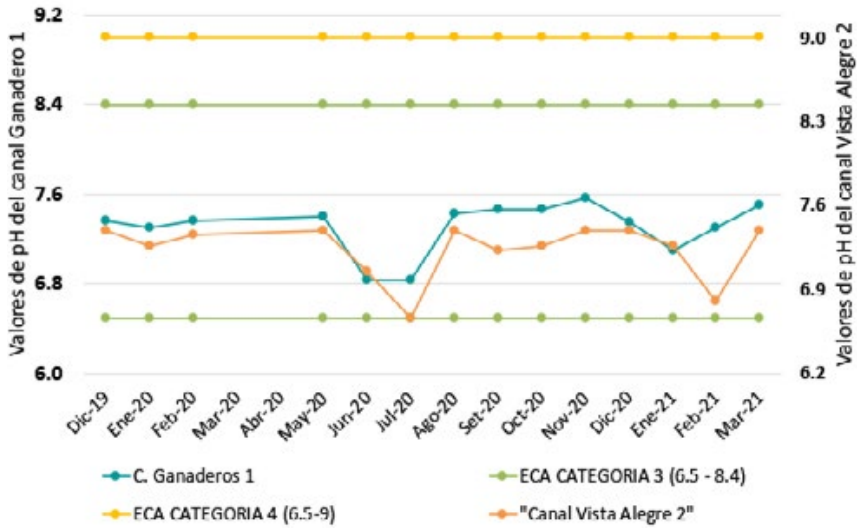

Figura 3. Valores de pH en el canal Ganaderos 1 en el periodo 2019-2021.

6

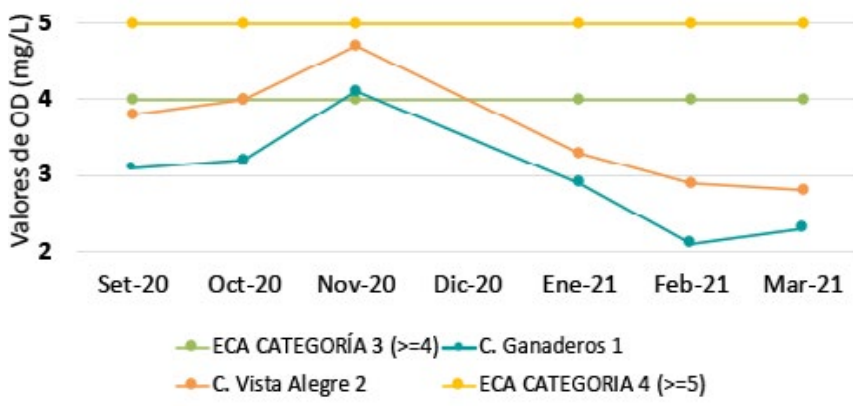

Figura 4. Valores de oxígeno disuelto en el canal Ganaderos 1 en el periodo 2019-2021.

categorías 3 y 4 , lo que indica que el valor ideal es $\geq 4 \mathrm{mg} / \mathrm{L}$ $y \geq 5 \mathrm{mg} / \mathrm{L}$, respectivamente. La presencia del oxígeno disuelto es importante, ya que influye en la mayoría de los procesos vitales de los organismos bióticos que habitan en el ecosistema (Betancourt et al., 2008). La excepción ocurre en noviembre, cuando ambos canales cumplen el ECA para la categoría 3. De igual manera, en enero y febrero se observan valores bajos de OD, lo que podría deberse a la elevada temperatura en la temporada de verano; ello incide en que exista una mayor competencia de los organismos por el consumo de oxígeno para desarrollar sus procesos naturales (Morell et al., 2015).

Tabla 3. Resultados de los parámetros de metales tóxicos de los canales Ganaderos 1 y Vista Alegre 2.

\begin{tabular}{|c|c|c|c|c|c|c|c|}
\hline \multirow{3}{*}{ Parámetros } & \multicolumn{3}{|c|}{$\begin{array}{l}\text { Categoría 3: riego de vegetales y bebida de } \\
\text { animales }\end{array}$} & \multirow{2}{*}{$\begin{array}{c}\text { Categoría 4: } \\
\text { conservación del } \\
\text { ambiente acuático }\end{array}$} & \multirow{3}{*}{$\begin{array}{c}\text { Canal } \\
\text { Ganaderos } \\
1 \\
\\
\text { PM-01 }\end{array}$} & \multirow{3}{*}{$\begin{array}{c}\begin{array}{c}\text { Canal } \\
\text { Ganaderos } 1\end{array} \\
\text { PM-02 }\end{array}$} & \multirow{3}{*}{$\begin{array}{c}\text { Canal Vista } \\
\text { Alegre } 2 \\
\\
\text { PM-03 }\end{array}$} \\
\hline & \multicolumn{2}{|c|}{ D1 } & \multirow{2}{*}{$\begin{array}{c}\text { D2 } \\
\text { Bebida de } \\
\text { animales }\end{array}$} & & & & \\
\hline & $\begin{array}{c}\text { Agua para } \\
\text { riego no } \\
\text { restringido }\end{array}$ & $\begin{array}{c}\text { Agua } \\
\text { para riego } \\
\text { restringido }\end{array}$ & & Lagunas y lagos & & & \\
\hline Plomo (mg/L) & 0,05 & 0,05 & 0,05 & 0,001 & 0,002 & 0,003 & 0,001 \\
\hline Mercurio (mg/L) & 0,001 & 0,001 & 0,01 & 0,0001 & 0,0006 & 0,0002 & 0,0002 \\
\hline Cadmio (mg/L) & 0,01 & 0,01 & 0,05 & 0,004 & 0,00017 & 0,00015 & 0,00013 \\
\hline Arsénico (mg/L) & 0,1 & 0,1 & 0,2 & 0,01 & 0,0039 & 0,0035 & 0,0029 \\
\hline
\end{tabular}


Los valores del nitrato desde octubre de 2020 a marzo de 2021 sobrepasaron el límite establecido de $13 \mathrm{mg} / \mathrm{L}$ en la categoría 4 del ECA para agua. No obstante, sí cumplen el valor de $100 \mathrm{mg} / \mathrm{L}$ en la categoría 3 del ECA para agua, a excepción de noviembre de 2020, cuando lo sobrepasó con un valor de 106,3 mg/L (figura 5). La cercanía de los canales con la urbanización y las fábricas probablemente generan contaminación por residuos orgánicos, aguas residuales domésticas o industriales, ya que de ello depende el incremento del nitrato (Larios, 2009). Las altas concentraciones de nitrato al oxidarse producen nitritos y nitrato que consumen rápidamente el oxígeno disuelto, lo cual es perjudicial en el agua, ya que genera un proceso de eutrofización que pone en riesgo la supervivencia de la biota (Roldán y Ramírez, 2008). La consecuencia sería la pérdida de los servicios ecosistémicos de provisión que brindan los canales a las actividades diarias de la población y la sobrevivencia de la biota.

\section{Índice de calidad del agua}

Mediante la aplicación de la ecuación 1 del ICA-UWQI se determinó que la calidad del agua de los canales Ganaderos 1 y Vista Alegre 2 es marginal, ya que presenta un valor de 45,6 de una escala de 100 marginal (color naranja) (tabla 4). Ello indica que la calidad del agua presenta condiciones muy alejadas a las que debería tener para mantenerse entre aceptable y buena (Caho-Rodríguez y López-Barrera, 2017). Se observa que los valores obtenidos de oxígeno disuelto, $\mathrm{DBO}_{5}$, nitrato, fósforo y conductividad eléctrica presentan concentraciones que sobrepasan el límite establecido por la categoría 3 y 4 del ECA para agua. De tal manera que los servicios ecosistémicos que brinda los canales son influenciados por actividades de la población y fábricas aledañas.

\section{Conclusiones}

Este estudio permitió demostrar que la calidad del agua de los canales Ganaderos 1 y Vista Alegre 2 están influenciados por factores naturales (como la presencia de algas) y factores antropogénicos (como las descargas

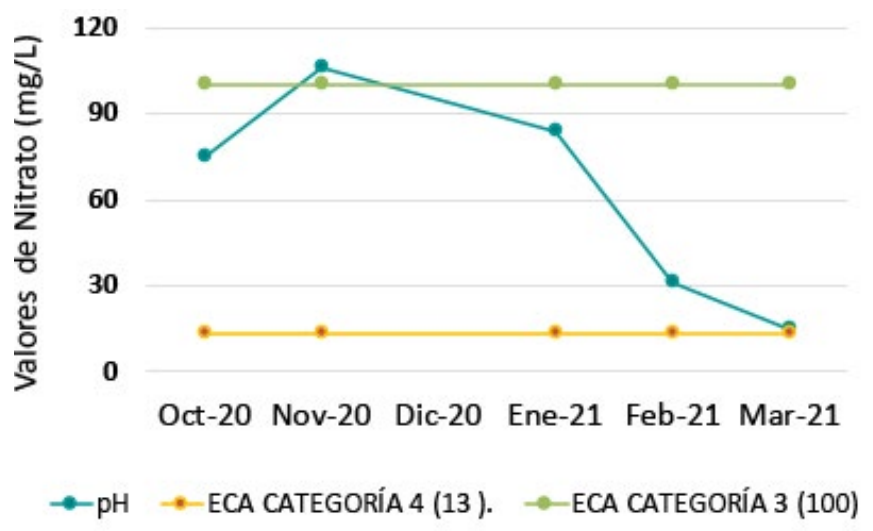

Figura 5. Valores de nitrato en el canal Ganaderos 1 en el periodo 20192021. de aguas residuales, la presencia de excretas de animales y los residuos sólidos). Los resultados del muestreo puntual demostraron que los parámetros nitrato, fósforo, $\mathrm{OD}, \mathrm{DBO}_{5}$ y Escherichia coli no cumplen con la categoría 3 y 4 del ECA para agua, que son un problema para el mantenimiento de servicios ecosistémicos de provisión, como agua para animales y abastecimiento al humedal y la población aledaña a los canales. Además, la presencia de Escherichia coli puede ocasionar problemas de salud en los pobladores. La presencia de fósforo y nitrato indicaría problemas de eutrofización. Los metales pesados no se vieron reflejados debido a la presencia de especies macrófitas, lo cual resulta ser un buen indicador para la calidad del agua, pero se recomendaría realizar otros análisis de sedimentación para descartar su presencia. En cuanto al análisis de data del periodo 2019-2021, los parámetros OD y nitrato demuestran que la calidad del agua no es óptima para la conservación de la biota y salud de los pobladores. Finalmente, el cálculo del ICA-UWQI nos permitió determinar una calidad de agua marginal, que se ve influenciada por los parámetros que excedieron su valor permisible. Se concluye que la calidad del agua no presenta óptimas condiciones para su uso, ya que podría ocasionar problemas a la salud y la conservación del medio.

\section{Recomendaciones}

Para una evaluación rigurosa de la calidad del agua de los canales Ganaderos 1 y Vista Alegre 2, se recomienda realizar una evaluación continua de los parámetros,

Tabla 4. Resultado del ICA de los canales Ganaderos 1 y Vista Alegre 2.

\begin{tabular}{|c|c|c|c|c|c|}
\hline \multirow{3}{*}{ Parámetro } & \multicolumn{3}{|c|}{ Subíndice (li) } & \multirow{3}{*}{$\begin{array}{l}\text { Peso } \\
\text { (Wi) }\end{array}$} & \multirow{3}{*}{ Subfinal } \\
\hline & $\begin{array}{c}\text { Canal } \\
\text { Ganaderos } \\
1\end{array}$ & $\begin{array}{c}\text { Canal } \\
\text { Ganaderos } \\
1\end{array}$ & $\begin{array}{c}\text { Canal } \\
\text { Vista } \\
\text { Alegre } \\
2\end{array}$ & & \\
\hline & PM-01 & PM-02 & PM-03 & & \\
\hline DBO & 0 & 0 & 0 & 0,06 & 0,00 \\
\hline OD & 10 & 20 & 17 & 0,11 & 1,77 \\
\hline $\mathrm{pH}$ & 100 & 100 & 100 & 0,03 & 2,90 \\
\hline Nitratos & 0 & 0 & 0 & 0,09 & 0,00 \\
\hline Fósforo & 28 & 46 & 86 & 0,06 & 3,04 \\
\hline Mercurio & 47 & 88 & 88 & 0,09 & 6,36 \\
\hline Cadmio & 100 & 100 & 100 & 0,09 & 8,60 \\
\hline Arsénico & 100 & 100 & 100 & 0,11 & 11,30 \\
\hline Coliformes T. & - & 100 & 100 & 0,11 & 11,40 \\
\hline Fluoruros & - & - & - & 0,09 & 0,09 \\
\hline Selenio & - & - & - & 0,09 & 0,09 \\
\hline Cianuro & - & - & - & 0,09 & 0,09 \\
\hline ICA-UWI & Marginal & & & 1,00 & 45,63 \\
\hline
\end{tabular}


en particular fisicoquímicos y microbiológicos, en todo el transecto, ya que la presencia de coliformes indica una alta probabilidad de encontrar varios tipos de bacterias del género Salmonella, que producen distintas infecciones. Asimismo, los valores altos de nitrato o fosfato provocarían eutrofización de los canales. Al no encontrarse valores significativos de metales tóxicos en el agua superficial y no haber investigaciones relacionadas con los metales tóxicos en sedimento en estos canales, se recomienda implementar un estudio basado en la acumulación de metales tóxicos en sedimento, en la flora que bordea los canales Ganaderos 1 y Vista Alegre 2 y en el humedal Pantanos de Villa.

\section{Contribución de autoría}

EE-A, CG-A y AG-S contribuyeron en el procesamiento de la información, el análisis de los resultados y la redacción del artículo. DCh-A y MAl-M contribuyeron en la gestión de la información, el procesamiento de la información, el análisis de los resultados y la redacción del artículo. Todos los autores aprobaron la versión final del texto.

\section{Fuente de financiamiento}

Este trabajo tuvo el soporte logístico de Prohvilla.

\section{Conflictos de interés}

Los autores declaran que no existe ningún conflicto de interés.

\section{Referencias bibliográficas}

Alarcón Corro, J. F. (2019). Aplicación de métodos de Índices de Calidad de Agua (ICA) en el río Rímac. Tesis para obtener el título profesional de ingeniero geógrafo. Universidad Nacional Mayor de San Marcos. Disponible en: https://cybertesis.unmsm.edu.pe/ handle/20.500.12672/12169

Álvarez Gutiérrez, C. (2016). Determinación analítica de detergentes en las aguas de los Pantanos de Villa. Tesis de licenciatura en Química. Pontificia Universidad Católica del Perú. Disponible en: http://tesis. pucp.edu.pe/repositorio/handle/20.500.12404/7570

Alvaro, S. S. (2020). Restauración de los canales eutrofizados de abastecimiento de agua en los Pantanos de Villa. Tesis de magíster en Desarrollo Ambiental. Pontificia Universidad Católica del Perú. Disponible en: http://hdl.handle.net/20.500.12404/17439

Aponte, H., Gonzales, S. y Gomez, A. (2020). «Impulsores de cambio en los humedales de América Latina: El caso de los humedales costeros de Lima». South Sustainability, 1, e023(1-5). Disponible en: https://doi.org/10.21142/SS-0102-2020-023

Aponte, H., Ramírez, W., Lértora, G., Vargas, R., Fernando, G., Carazas, N. y Liviac, R. (2015). «Incendios en los humedales de la costa central del Perú: una amenaza frecuente». Científica, 12(1), pp. 70-81. Disponible en: https://revistas.cientifica.edu.pe/index.php/ cientifica/article/view/166/187

Autoridad de Salud de Oregon. (2011). «Nitrato». Disponible en: https://www.oregon.gov/oha/PH/HealthyEnvironments/ DrinkingWater/Monitoring/Documents/health/no3-sp.pdf
Autoridad Nacional del Agua. (2016). Protocolo nacional para el monitoreo de la calidad de los recursos hídricos superficiales. Autoridad Nacional del Agua. Disponible en: https://www.ana.gob. pe/publicaciones/protocolo-nacional-para-el-monitoreo-de-lacalidad-de-los-recursos-hidricos-0

Ávila De Navia, S. L. y Estupiñán-Torres, S. M. (2006). «Calidad bacteriológica del agua del Humedal de Jaboque, Bogotá, Colombia. Caldasia, 28(1), pp. 67-78. Disponible en: http://www.scielo.org.co/ pdf/cal/v28n1/v28n1a7.pdf

Baquero, C. y Carrera, C. (2019). Evaluación de parámetros fisicoquímicos de aguas mieles de cacao en humedales artificiales con especies macrófitas, Tarapoto, 2019. Tesis para obtener el título profesional de ingeniero ambiental. Universidad César Vallejo. Disponible en: https://repositorio.ucv.edu.pe/handle/20.500.12692/41536

Bernot, M. y Wilson, K. (2012). «Spatial and temporal variation of dissolved oxygen and ecosystem energetics in Devils Hole, Nevada». Western North American Naturalist, 72(3), pp. 265-275. Disponible en: https://doi.org/10.3398/064.072.0301

Betancourt, C., Suárez, R. y Toledo, L. (2008). «Patrones de distribución temporal de algunas variables físicas y químicas en el embalse Paso Bonito, Cienfuegos, Cuba». Limnetica, 28(1), pp. 23-34. Disponible en: http://www.limnologia.net/documentos/limnetica/limnetica28-1-p-23.pdf

Bolaños, J., Cordero, G. y Segura, G. (2017). «Determinación de nitritos, nitratos, sulfatos y fosfatos en agua potable como indicadores de contaminación ocasionada por el hombre, en dos cantones de Alajuela (Costa Rica)». Tecnología en Marcha, 30(4), pp. 15-17. Disponible en: https://www.scielo.sa.cr/pdf/tem/v30n4/03793982-tem-30-04-15.pdf

Boyacioglu, H. (2007). «Desarrollo de un índice de calidad del agua basado en un esquema de clasificación europeo». Agua SA, 33(1), pp. 101-106.

Caho-Rodríguez, C. y López-Barrera, E. A. (2017). «Determinación del Índice de Calidad de Agua para el sector occidental del humedal Torca-Guaymaral empleando las metodologías UWQI y CWQI». Lasallista, 12(2), pp. 35-49. Disponible en: http://www.scielo.org.co/ $\mathrm{pdf} / \mathrm{pml} / \mathrm{v12n2/1909-0455-pml-12-02-00035.pdf}$

Castro, M., Almeida, J., Ferrer, J. y Díaz, D. (2014). «Indicadores de la calidad del agua: evolución y tendencias a nivel global». Ciencias Biológicas, 10(17), pp. 111-124. Disponible en: https://revistas.ucc.edu. co/index.php/in/article/view/811/770

Cervantes, F., Pérez, J. y Gómez, J. (2000). «Avances en la eliminación biológica del nitrógeno de las aguas residuales». Revista Latinoamericana de Microbiología, 42(2), pp. 73-82. Disponible en: https://www.medigraphic.com/pdfs/lamicro/mi-2000/mi002e.pdf

Combariza Bayona, D. (2009). Contaminación por metales pesados en el embalse del Muña y su relación con los niveles en sangre de plomo, mercurio y cadmio y alteraciones de salud en los habitantes del municipio de Sibaté (Cundinamarca). Tesis de magíster en Toxicología. Universidad Nacional de Colombia. Disponible en: https://repositorio.unal.edu.co/bitstream/handle/ unal/70168/597588.2009.pdf? sequence=1\&isAllowed $=y$

Correa, J. (2014). «Calidad del agua en humedales del plano de inundación del río Atrato». Ciencias Ambientales y Sostenibilidad CAS, 1(1), pp. 93-109. Disponible en: https://revistas.udea.edu.co/ index.php/CAA/article/view/16917/16758

Delgadillo, A., González, C., Prieto, F., Villagómez, J. y Acevedo, O. (2011). «Fitorremediación: una alternativa para eliminar la contaminación». Tropical and Subtropical Agroecosystems, 14(2), pp. 597-612. Disponible en http:// www.scielo.org. $\mathrm{mx} /$ scielo.php? script=sci_arttext\&pid=S187004622011000200002\&lng=es\&tlng=es 
Domínguez, A. L. (2001). «Evaluación de la depuración de las aguas residuales provenientes de un sistema de tratamiento combinado de laguna de estabilización y laguna con Jacinto de agua». Actualidades Biológicas, 23(74), pp. 75-82. Disponible en: https://revistas.udea. edu.co/index.php/actbio/article/view/329622/20786030

Gobierno del Perú (2018). Decreto Supremo 004. Aprueban Estándares de Calidad Ambiental (ECA) para Agua y establecen Disposiciones Complementarias. 7 de junio de 2017. Ministerio del Ambiente. Disponible en: https://sinia.minam.gob.pe/normas/apruebanestandares-calidad-ambiental-eca-agua-establecen-disposiciones

Espitia, N. (2014). «Determinación de metales pesados en sedimentos superficiales en cuerpos de agua del canal del dique en las poblaciones de Gambote y Soplaviento (Bolívar)». Revista del Instituto de Investigación, FIGMMG-UNMSM, 17(34), pp. 91-100. Disponible en: https://revistasinvestigacion.unmsm.edu.pe/index. php/iigeo/article/view/11389/10227

Fajardo, N., Solís, H. y Gil, F. (2018). «Determinación de metales pesados en los cuerpos de agua del Área de Conservación Regional Humedales de Ventanilla, Región Callao, Perú». Revista del Instituto de Investigación, FIGMMG-UNMSM. Disponible en: https:// revistasinvestigacion.unmsm.edu.pe/index.php/iigeo/article/ view/14177/12490

Fernández Gonzales, J., De Miguel Beascochea, E., De Miguel Muñoz, J. y Curt Fernández de la Mora, D. (2005). Fitodepuración. Filtros de macrófitas en flotación. Proyecto Life. Disponible en: https:// fundacionglobalnature.org/wp-content/uploads/2020/01/manual_ fitodepuracion.pdf

García, J., Rousseau, D., Morató, J., Lesage, E., Matamoros, V. y Bayona, J. (2010). «Contaminant removal processes in subsurface-flow constructed wetlands: A review». Critical Reviews in Environmental Science and Technology, 40(7), pp. 561-611. Disponible en: https:// www.tandfonline.com/doi/abs/10.1080/10643380802471076

Gaxiola Castro, G. y Durazo, R. (Eds.). (2010). Dinámica del ecosistema pelágico frente a Baja California, 19972007. Disponible en: https://books.google.com.pe/ books? id=ji3FfrrZ1cYC\&printsec $=$ frontcover \&hl=es\&source $=$ gbs_ ge_summary_r\&cad $=0 \% 23 v \% 3$ Donepage $\& q \& f=f a l s e \# v=$ onepage\&q\&f=false

Gil-Marín, J. A., Vizcaino, C. y Montaño-Mata, N. J. (2018). «Evaluación de la calidad del agua superficial utilizando el índice de calidad del agua (ICA). Caso de estudio: Cuenca del Río Guarapiche, Monagas, Venezuela». Anales Científicos, 79(1), pp. 111-119. Disponible en: https://revistas.lamolina.edu.pe/index.php/acu/article/view/1146/ pdf 77

Guillén, V., Teck, H., Kohlmann, B. y Yeomans, J. (2012). «Microorganismos como bioindicadores de la calidad del agua». Tierra Tropical: Sostenibilidad, Ambiente y Sociedad, 8(1), pp. 65-93.

Haustein, E., González, M., Peña-Cortés, F. y Muñoz-Pedreros, A. (2005). «Diversidad vegetal en humedales costeros de la Región de La Araucanía». En: Historia, biodiversidad y ecología de los bosques costeros de Chile (pp. 197-205). Editorial Universitaria. Disponible en: http://www.ceachile.cl/publicaciones/2005.\%20 Vegetaci\%F3n\%20Cordillera\%20Hauenstein\%20et\%20al.pdf

Herrera, M. (2018). Eficiencia de Eichornia spp y Lemna spp nativas en humedales artificiales en la remoción de sulfonato de alquilbenceno lineal de los detergentes presentes en aguas residuales domésticas, Moyobamba. 2017. Tesis para obtener el título profesional de ingeniero ambiental. Universidad Nacional de San Martín. Disponible en: https://repositorio.unsm.edu.pe/handle/11458/2790

Hill, R. W. (1980). Fisiología animal comparada. Reverté. Disponible en: https://books.google.com.pe/books?id= eMYiEAAAQBAJ\&printsec $=$ frontcover \&dq $=\% 22 \mathrm{Fisiolog} \%$ C3\%ADa+animal+comparada $\% 22 \& \mathrm{hl}=$ es\&sa=X\&redir_esc $=\mathrm{y} \# \mathrm{v}=$ onepage\&q=\%22Fisiolog\%C3\%ADa\%20animal\%20comparada\% $22 \& f=f a l s e$
Huaman-Vilca, S., Lucen-Espinoza, M., Paredes-Vite, M. y Changanaqui Alfaro, D. (2020). «Evaluación de la calidad del agua de la laguna Marvilla en los Pantanos de Villa (Lima, Perú)». Universidad Científica del Sur (Lima, Perú), 1(2), pp. 1-8. Disponible en: https://revistas.cientifica.edu.pe/index.php/southsustainability/ article/view/779/74 7

Ionus, O. (2010). Water Quality Index - assessment method of the Motru River. Water quality (Oltenia, Romania). Annals of the University of Craiova, 13 (new series) (10), pp. 74-82.

Instituto de Hidrología, Meteorología y Estudios Ambientales, IDEAM. (2011). Hoja metodológica del indicador Índice de calidad del agua (Versión 1,00). Disponible en: http://www.ideam.gov.co/ documents/24155/125494/53-3.22 HM IACAL 3 Fl.pdf/0b603e3d7b31-4b31-9e8e-71ce9bbdf2e5

Larios, L. (2009). Contaminación del agua por nitratos: significación sanitaria. Revista Archivo Medico de Camagüey, 13(2). Disponible en: http://scielo.sld.cu/scielo.php?script=sci_arttext\&pid=S102502552009000200017

Mara, D. (2003). Domestic wastewater treatment in developing countries. Routledge. Disponible en: https://www.taylorfrancis.com/books/ mono/10.4324/9781849771023/domestic-wastewater-treatmentdeveloping-countries-duncan-mara

Martelo, J. y Lara, J. (2012). «Macrófitas flotantes en el tratamiento de aguas residuales: una revisión del estado de arte». Ingeniería y Ciencia, 8(15), pp. 221-243. Disponible en: http://www.scielo.org.co/ pdf/ince/v8n15/v8n15a11.pdf

Martinez Durán, M. E., Pacheco, O. y Quijada Bonilla, H. (2016). Protocolo de vigilancia en salud pública. Enfermedades transmitidas por alimentos. (2. - ed.). Instituto Nacional de Salud.

Merino, F. y Droste, H. (1983). «Adaptación del Guppy Lebistes reticulatus al ambiente II. La adaptación fisiológica-metabólica». Actualidades Biológicas, 12(34.4), pp. 68-76. Disponible en: https://revistas.udea. edu.co/index.php/actbio/article/view/330312/20786584

Morell, A., Bergues, P. y Portuondo, E. (2015). «Valoración de los parámetros físicos-químicos de las aguas del río San Juan en los periodos húmedo y seco de 2014». Ciencia en su PC, 1, pp. 1-12. Disponible en: https://www.redalyc.org/articulo. oa?id=181338814001

Nava-Ruiz, C. y Méndez-Armenta, M. (2011). «Efectos neurotóxicos de metales pesados (cadmio, plomo, arsénico y talio)». Archivos de Neurociencias, 16(3), pp. 140-147. Disponible en: https://www. medigraphic.com/pdfs/arcneu/ane-2011/ane113f.pdf

Pérez, R., Riveiro, F., Jiménez, M., Manganiello, L., Vega, C., Covad, R. y Moreno, J. (2017). Water quality assessment in a Caribbean saltwater wetland». Revista Ingeniería UC, 24(3), pp. 417-427. Disponible en: https://www.researchgate.net/publication/325812788 Water quality_assessment_in_a_Caribbean_saltwater_wetland

Prohvilla, Autoridad Municipal de Los Pantanos de Villa. (2020). «Monitoreo y evaluación de las especies de flora, Lima». OICDP.

Pulido, V. y Bermúdez, L. (2018). «Estado actual de la conservación de los hábitats de los Pantanos de Villa, Lima, Perú». Arnaldoa, 25(2), pp. 679-702. Disponible en: http://www.scielo.org.pe/pdf/arnal/ v25n2/a19v25n2.pdf

Quiroz Fernández, L. S., Izquierdo Kulich, E. y Menéndez Gutiérrez, C. (2017). «Aplicación del índice de calidad de agua en el río Portoviejo, Ecuador». Ingeniería Hidráulica y Ambiental, XXXVIII(3), pp. 41-51. Disponible en: http://scielo.sld.cu/pdf/riha/v38n3/riha04317.pdf

Raffo Lecca, E. y Ruiz Lizama, E. (2014). «Caracterización de las aguas residuales y la demanda bioquímica de oxígeno». Industrial Data, 17(1), pp. 71-80. Disponible en: https://revistasinvestigacion.unmsm. edu.pe/index.php/idata/article/view/12035/10751 
Raul, O. (2011). Actitudes hacia el medio ambiente en un grupo de pobladores de las inmediaciones de los Pantanos de Villa. Tesis de licenciatura en Psicología con mención en Psicología Social. Pontificia Universidad Católica del Perú. Disponible en: https://tesis. pucp.edu.pe/repositorio/bitstream/handle/20.500.12404/799/ OTERO_ROOSE_RAUL_ACTITUDES_MEDIO_AMBIENTE. pdf? sequence $=1 \&$ is Allowed $=y$

Riđanović, L., Riđanović, S., Jurica, D. y Spasojević, P. (2010). «Evaluation of water temperature and dissolved oxygen regimes in River Neretva». Orhid. Disponible en: https://balwois.com/wpcontent/uploads/old proc/ffp-1520.pdf

Ríos-Tobón, S., Agudelo-Cadavid, R. y Gutiérrez-Builes, L. (2017). «Patógenos e indicadores microbiológicos del agua para consumo». Revista Facultad Nacional de Salud Pública, 35(2), pp. 236-247. Disponible en: http://www.scielo.org.co/pdf/rfnsp/v35n2/0120386X-rfnsp-35-02-00236.pdf

Rock, C. y Rivera, B. (1914). «La calidad del agua, E. coli y su salud». College of Agriculture, pp. 1-5. Disponible en: https://extension. arizona.edu/sites/extension.arizona.edu/files/pubs/az1624s.pdf

Rodríguez, R., Retamozo-Chavez, R., Aponte, H. y Valdivia, E. (2017). «Evaluación microbiológica de un cuerpo de agua del ACR Humedales». Ecología Aplicada, 16(1), pp. 15-21. Disponible en: http://www.scielo.org.pe/pdf/ecol/v16n1/a03v16n1.pdf

Roldán, G. y Ramírez, J. (2008). Fundamentos de limnología tropical. (2. a ed.). Universidad de Antioquia. Disponible en: https://www. untumbes.edu.pe/vcs/biblioteca/document/varioslibros/0742.\%20 Fundamentos\%20de\%20limnolog\%C3\%ADa\%20neotropical.pdf

Romero Ledezma, K. P. (2009). «Contaminación por metales pesados». Revista Científica Ciencia Médica, 12(1), pp. 45-46. Disponible en: https://www.redalyc.org/pdf/4260/426041218013.pdf

Ruiz Sánchez, B. B., Rodríguez, R. e lpanaqué, J. (2013). «Determinación de la acumulación de metales tóxicos en agua, sedimento y biota del humedal El Paraíso». Infinitum, 3(2), pp. 33-39. Disponible en: https://revistas.unjfsc.edu.pe/index.php/INFINITUM/article/ view/379/349
Sánchez-Viveros, G. D., Gonzales-Mendoza, D., Alarcon, A. y FerreraCerrato, R. (2010). «Cooper effects on photosynthetic activity and membrane leakage of Azolla filiculoides and A. caroliniana». International Journal of Agriculture and Biology, 12(3), pp. 365-368.

Sedano-Chiroque, F. L., Rojas-Milian, C. y Vela-Ruiz, J. M. (2020). «COVID-19 desde la perspectiva de la prevención primaria». Revista de la Facultad de Medicina Humana de la Universidad Ricardo Palma, 20(3), pp. 494-501. Disponible en: http://www.scielo.org.pe/pdf/ rfmh/v20n3/2308-0531-rfmh-20-03-494.pdf

Silva Burga, J. (2004). Evaluación y rediseño del sistema de lagunas de estabilización de la universidad de Piura. Tesis para obtener el título profesional de ingeniero civil. Universidad de Piura. Disponible en: https://pirhua.udep.edu.pe/bitstream/handle/11042/1189/ICI_119. pdf

Solano Carrion, A. L. (2019). Comparación de la eficiencia de Pistia stratiotes y Azolla filiculoides para mejorar la calidad del agua residual del dren 4000. Tesis para obtener el título profesional de ingeniera ambiental. Universidad César Vallejo. Disponible en: https:// repositorio.ucv.edu.pe/bitstream/handle/20.500.12692/35251/ Solano_CAL.pdf? sequence $=1 \&$ isAllowed $=y$

Teixeira Correia, G., Sánchez Ortiz, I. A., Gebara, D., Dall'Aglio Sobrinho, M. y Matsumoto, T. (2013). «Remoción de fósforo de diferentes aguas residuales en reactores aeróbios de lecho fluidizado trifásico con circulación interna». Revista Facultad de Ingeniería Universidad de Antioquia, 67, pp. 172-182. Disponible en: https:// www.redalyc.org/articulo.oa?id=43029146015

Torres, P., Hernán Cruz, C. y Patriño, P. (2009). «Índices de calidad de agua en fuentes superficiales utilizadas en la producción de agua para consumo humano. una revisión crítica». Ingenierías Universidad de Medellín, 8(15), pp. 79-94. Disponible en: http://www.scielo.org. co/pdf/rium/v8n15s1/v8n15s1a09.pdf

World Health Organization, WHO. (2008). Guidelines for DrinkingWater Quality. (1. a ed.). World Health Organization. Disponible en: https://www.joinforwater.ngo/sites/default/files/library_ assets/351_WHO_E13_guidelines_drinking-water.pdf 